\title{
Editorial
}

\section{Time is the Enemy}

DOI: $10.3766 /$ jaaa.30.2.1

$\mathrm{I}$ $\mathrm{t}$ has been demonstrated many times using behavioral techniques that auditory perception suffers when listeners must process auditory signals at faster compared to normal rates. It is the effect that "aging" has on auditory reception and perception that can at least, in part, explain poorer performance by elder subjects on auditory perceptual tasks. In this month's JAAA, McCullagh and Shinn have engaged two groups of adults in a classical P300 "oddball" paradigm. The two groups of subjects were younger (mean age 21 years) and older (mean age 66 years). For those of you who are unfamiliar with the "oddball" paradigm, the listener is presented (usually binaurally) with two tones, one that is lower in pitch (e.g., $500 \mathrm{~Hz}$ ) that occurs frequently (e.g., with a probability of occurrence of $85 \%$ ) and another that is higher in pitch (e.g., $1000 \mathrm{~Hz}$ ) that occurs infrequently (e.g., with a probability of occurrence of $15 \%$ ). The listener's task is to detect when the infrequently occurring tone (the "oddball") occurs. Some type of response usually is required of the subject that indicates they have identified the infrequent tone. The response might be a button press or the subject might be asked to count silently the infrequently occurring tone.

In the current article, listeners were asked to count the number of oddball tones and report the total to the investigator. When brain activity is recorded during that paradigm, a number of evoked responses can be recorded. These responses included the exogenous

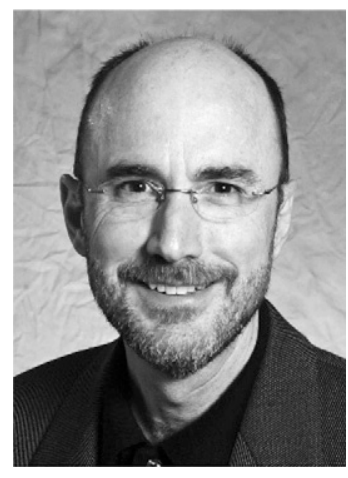

(i.e., the obligatory) N1 and P2 responses. Additionally, there is a second negativity (N2a representing the pre-conscious recognition by the brain that the oddball has occurred) and that is followed by the positivity called the P300 (representing the conscious recognition that a significant event has occurred). It is referred to as P300 because using the simplest paradigm the late positivity shows a median latency of $\sim 300 \mathrm{~ms}$. There are a number of variables that can influence the latency and amplitude of P300 including the difficulty of the perceptual task. That it should take longer to identify the target signal when the perceptual task is made more difficult makes sense, doesn't it?

The investigators assessed the P300 (latency and amplitude) in young adults and older adults. They varied task difficulty by systematically worsening the signal to noise ratio from $+20 \mathrm{~dB}$ to $+10 \mathrm{~dB}$ to $0 \mathrm{~dB}$.

You will have to read the article to obtain the details but using the endogenous P300 event related potential the investigators reported that, in general, P300 latencies were significantly longer in the older group. The editors invite you to read this interesting article in the February issue of $\boldsymbol{J A A A}$.

Gary P. Jacobson, Ph.D. Editor-in-Chief, Journal of the American Academy of Audiology 